\title{
Microglia in prion diseases
}

\author{
Adriano Aguzzi and Caihong Zhu \\ Institute of Neuropathology, University Hospital Zurich, Zurich, Switzerland.
}

\begin{abstract}
Prion diseases are a group of progressive and fatal neurodegenerative disorders characterized by deposition of scrapie prion protein $(\mathrm{PrPs})$ in the CNS. This deposition is accompanied by neuronal loss, spongiform change, astrogliosis, and conspicuous microglial activation. Here, we argue that microglia play an overall neuroprotective role in prion pathogenesis. Several microglia-related molecules, such as Toll-like receptors (TLRs), the complement system, cytokines, chemokines, inflammatory regulators, and phagocytosis mediators, are involved in prion pathogenesis. However, the molecular mechanisms underlying the microglial response to prion infection are largely unknown. Consequently, we lack a comprehensive understanding of the regulatory network of microglial activation. On the positive side, recent findings suggest that therapeutic strategies modulating microglial activation and function may have merit in prion disease. Moreover, studies on the role of microglia in prion disease could deepen our understanding of neuroinflammation in a broad range of neurodegenerative disorders.
\end{abstract}

\section{Introduction}

Prion diseases, also known as transmissible spongiform encephalopathies, are a group of progressive and fatal neurodegenerative disorders affecting humans (such as Creutzfeldt-Jakob disease, kuru, Gerstmann-Sträussler-Scheinker disease, and fatal familiar insomnia) and several other mammals (such as scrapie in sheep and goats, bovine spongiform encephalopathy in cattle, and chronic wasting disease in deer, moose, and elk) $(1,2)$. Prion diseases are characterized by the deposition of scrapie prion protein $\left(\mathrm{PrP}^{\mathrm{Sc}}\right)$, a misfolded form of cellular prion protein $\left(\operatorname{Pr}^{\mathrm{C}}\right)$, in the CNS. This deposition is accompanied by neuronal loss, spongiform change, and gliosis. The etiology of prion diseases can be sporadic, heritable, or transmissible between individuals, making it unique among neurodegenerative disorders. The transmission of prions caused an epidemic of kuru in Papua New Guinea in the 1950s and 1960s and a bovine spongiform encephalopathy (also known as mad cow disease) crisis in the 1980s and 1990s $(3,4)$. Of even greater concern is the observation that bovine spongiform encephalopathy has been transmitted to humans by the consumption of prion-contaminated meat, causing hundreds of cases of variant Creutzfeldt-Jakob disease (5). According to the proteinonly hypothesis of prion disease, the infectious agent consists of mainly, if not solely, $\operatorname{PrP}^{\mathrm{Sc}}(6)$. $\mathrm{PrP}^{\mathrm{Sc}}$ acts as a propagon to recruit and convert $\mathrm{PrP}^{\mathrm{C}}$ to its own conformation through a selfperpetuating reaction. Like most other neurodegenerative disorders, prion diseases are incurable thus far.

Microglia are resident myeloid cells of the CNS that originate from $\mathrm{c}-\mathrm{Kit}^{+}$erythromyeloid precursors in the yolk sac and migrate to the developing neural tube for maturation, following a stepwise developmental program (7-11). Microglia play crucial roles in early development of the brain as well as the maintenance of brain homeostasis throughout life. Impairment of microglial functions

Conflict of interest: The authors have declared that no conflict of interest exists. Reference information: J Clin Invest. 2017;127(9):3230-3239.

https://doi.org/10.1172/JCI90605. can lead to severe pathological outcomes. For instance, disruption of the homeobox gene Hoxb8 in microglia results in a phenotype of excessive grooming in mice, mimicking trichotillomania in humans, an obsessive-compulsive spectrum disorder (12). Deficiency of the fractalkine receptor (CX3CR1), which is exclusively expressed by microglia in the CNS, leads to a transient reduction of microglia number in the developing brain and delayed synaptic pruning and circuit maturation (13). Additionally, microglia have been reported to play an important role in learning and memory by promoting learning-dependent synapse formation through brain-derived neurotrophic factor (BDNF) signaling (14).

As the primary immune cells and phagocytes in the CNS, microglia are highly dynamic and continuously patrol the brain parenchyma to engulf and clear apoptotic cells and cell debris (15). Microglia are also considered the most sensitive sensors of brain pathology. Under stimuli such as brain injury, microbial infection, or neurodegeneration, microglia can be activated, leading to morphological and molecular changes and cytokine release (16). Depending on the type of stimuli and pathological context, as well as the signals from the surrounding microenvironment, microglia can take on different activation states and become either neuroprotective or neurotoxic (17). Activated microglia can exert detrimental effects by producing proinflammatory mediators, such as TNF- $\alpha$, IL-1 $\beta$, IL-6, NO, and ROS (18). Alternatively, activated microglia can mediate beneficial effects through the release of antiinflammatory factors, such as IL-4, IL-10, and TGF- $\beta$ (19).

Microglial activation represents one of the hallmarks of many neurodegenerative diseases (20). However, prion disease is often associated with dramatic and conspicuous microglial activation (16). Study of prion diseases has benefited from mouse models of prion infection. Upon infection, WT mice can recapitulate most pathological features of prion diseases, including microglial activation. In this Review, we discuss microglial activation and cytokine release during prion disease progression and the overall role of microglia in prion pathogenesis, highlight the molecular mechanisms underlying the microglial response to prion infection, and 


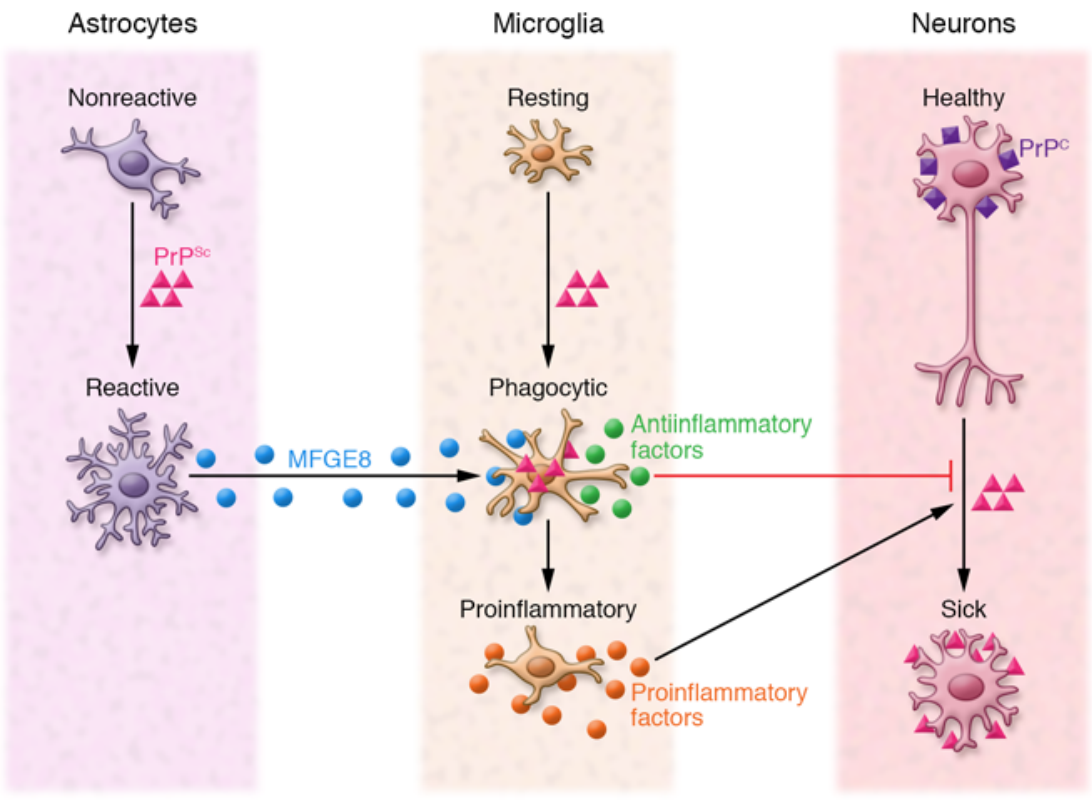

Figure 1. Microglial activation in prion disease. At an early stage of prion infection and $\mathrm{PrP}$ aggregation, microglia are activated by $\mathrm{PrP}^{\mathrm{Sc}}$. Microglia then acquire a phagocytic phenotype and produce several antiinflammatory cytokines such as IL-4, IL-10, and TCF- $\beta$. The phagocytosis of prions by microglia is facilitated by the astrocyte-derived opsonin MFGE8. However, over time, the clearance of prion becomes insufficient, and sustained $\mathrm{PrP}^{\mathrm{Sc}}$ accumulation leads to neuronal damage. This damage may trigger microglia to switch to a proinflammatory phenotype with secretion of TNF- $\alpha$, IL-1 $1 \beta$, and IL-6. Such inflammatory cytokines may also convert astrocytes to a deleterious $\mathrm{A} 1$ phenotype.

review the role of microglia-related molecules in prion pathogenesis. In view of recent advances in understanding the role of microglia in prion diseases, we discuss potential therapeutic targets for prion diseases based on modulation of microglial activation.

\section{Microglial activation and cytokine release in prion diseases}

Prion infection typically does not trigger an overt adaptive immune response in the periphery. Classical leukocyte infiltration and inflammatory response are usually absent in prion-infected brains (21). Neuronal loss and astrogliosis have been well documented during early studies of prion neuropathology; however, microglial activation has received less attention. By immunohistochemical analysis, microglia were first observed to be closely associated with plaques in Creutzfeldt-Jakob disease, GerstmannSträussler-Scheinker, and kuru (22-24). Compared with nonprion controls, immunohistochemical staining with microglial markers revealed markedly increased microglia in Creutzfeldt-Jakob disease and animal models of prion diseases $(25,26)$. The morphology of the microglia changed to an activated state, providing evidence of a microglial response in prion diseases.

Time course studies revealed that microglial activation appeared at an early phase of prion disease, before neuronal loss, spongiform change, and the onset of clinical signs (27-29), indicating that microglial activation might contribute to neurodegeneration as opposed to occurring merely as a secondary effect. Moreover, the distribution of microglial response correlates with spongiform change and astrogliosis, implying a close association between microglial activation and prion-induced neurodegeneration. Interestingly, microglial activation may vary between different human prion disease subtypes, since Western blots and immunohistochemical assays have demonstrated unaltered microglia in fatal familiar insomnia and genetic Creutzfeldt-Jakob disease (G114V) patients (30).

Along with microglial activation, expression of proinflammatory cytokines such as TNF- $\alpha$, IL- $1 \alpha$, and IL- $1 \beta$ is significantly increased in brains of prion-infected mice and Creutzfeldt-Jakob disease patients (31-33). Time course studies in mouse models of prion infection found that the temporal pattern of cytokine upregulation correlated with the onset and development of molecular and clinical prion pathology (31). The induction of cytokines in prion-infected mice was predominantly observed in brain regions showing neurodegeneration and spongiform change (34), suggesting that cytokines may play an important role in prion pathogenesis. Intriguingly, the induction of cytokine expression in mouse models of prion infection depends on prion strain/mouse genetic background combinations, since ME7infected C57BL/6J mice failed to show any significant change in cytokine profiling (35).

Microglia activation-associated cytokine release may not always be proinflammatory. In different rodent models of prion diseases, the antiinflammatory cytokine TGF- $\beta$ was also progressively upregulated $(36,37)$; however, the temporal patterns and extent of upregulation varied substantially among models, implying that the induction of cytokine differs according to prion strain and species infected (36). Similarly, levels of the antiinflammatory cytokines IL- 4 and IL-10 were also increased in the cerebrospinal fluid of Creutzfeldt-Jakob disease patients (38).

A comprehensive study of microglial response in prion disease was performed by whole transcriptome profiling of prion-infected mouse brains. This study revealed that the genes upregulated after prion infection, regardless of the prion strain or mouse genetic background, are expressed predominantly by activated microglia (39), contributing to a growing recognition of the importance of microglia in prion pathogenesis. Analysis of microglia isolated from prion-infected mice demonstrated that, in addition to an upregulation of genes associated with metabolic and respiratory stress, a disease-specific, highly proinflammatory profile was observed (39). Another transcriptomic analysis of various brain regions in the presence or absence of neurodegeneration revealed that there are at least two distinct microglial responses in prion-infected brains: a homeostatic response across all brain regions and an innate immune response restricted to sites of neurodegeneration (40).

In conclusion, microglial activation during prion disease progression is a complex and multistep process, with activated microglia comprising a heterogeneous population with diverse functions. At early stages of prion infection, microglia respond to $\mathrm{PrP}^{\mathrm{Sc}}$ deposits and consequently increase their phagocytic capacity 


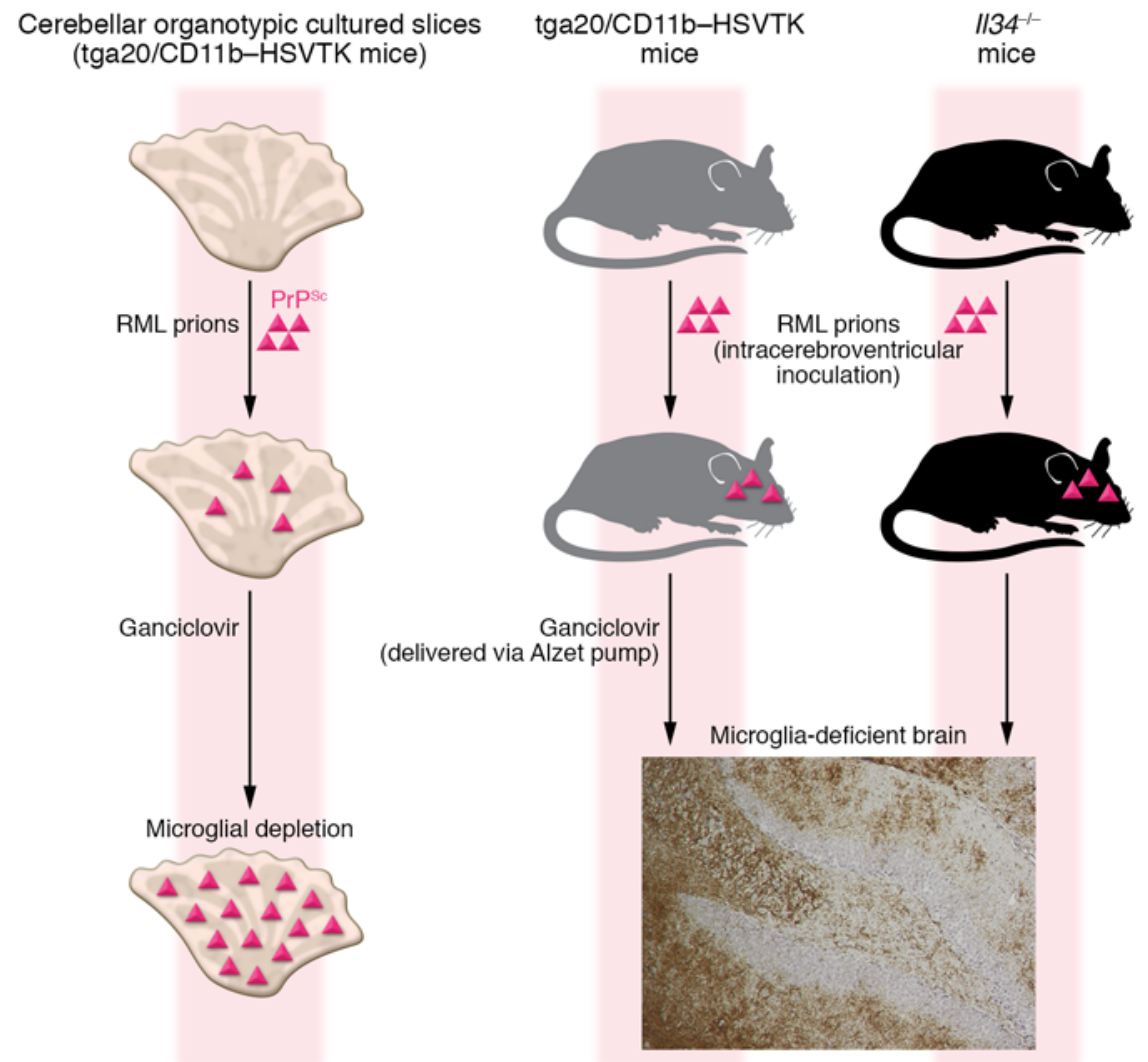

Enhanced prion deposition and prion-induced neurodegeneration
Figure 2. Microglia play a neuroprotective role in prion pathogenesis. Cerebellar organotypic cultured slices prepared from tga20/CD11b-HSVTK transgenic mice were infected with prions, followed by ganciclovir treatment. This leads to an efficient depletion of microglia and, consequently, enhanced $\mathrm{PrP}^{\mathrm{Sc}}$ deposition and neurotoxicity. Tga20/CD11b-HSVTK transgenic mice were first intracerebrally inoculated with prions. Ganciclovir was then delivered to the brain through osmotic pumps. As a result, prion pathogenesis was greatly enhanced. Similarly, when $1 / 34^{-/-}$mice (which contain fewer microglia than WT mice) were intracerebrally inoculated with prions, PrPsc accumulation was augmented and disease progression was accelerated. These results provide evidence for an overall neuroprotective role of microglia in prion pathogenesis. to remove $\operatorname{PrP}^{\mathrm{Sc}}$. However, this phagocytosis is insufficient, and sustained $\mathrm{PrP}^{\mathrm{Sc}}$ accumulation leads to neuronal damage, which could in turn trigger the microglia to switch to a proinflammatory phenotype and exert detrimental effects in the brain (Figure 1).

\section{Overall role of microglia in prion pathogenesis}

Although microglial activation is now well established and is considered a hallmark of prion neuropathology, the overall role of microglia in prion pathogenesis has remained contentious. Microglia are a highly dynamic and motile cell population in the CNS. In prion-infected mice, microglia are observed surrounding $\mathrm{PrP}^{\mathrm{Sc}}$ deposits and intracellular $\operatorname{PrP}^{\mathrm{Sc}}$ can be detected within microglia $(22-24,41,42)$. The presence of prion infectivity in microglia suggests that microglia might help disseminate prion within the brain. Activated microglia in prion-infected brain produce proinflammatory cytokines that may conceivably contribute to disease progression. However, microglia are professional phagocytes that can engulf and degrade prions, thereby decreasing prion load and slowing down prion progression.

A study using the prion protein-derived peptide $\mathrm{PrP}^{106-126}$ to treat primary cell cultures demonstrated that microglia are required for $\mathrm{PrP}^{106-126}$-induced cytotoxicity in primary neurons, suggesting that in this context microglia are an important mediator of neuronal death (43). However, PrP ${ }^{106-126}$ treatment is fundamentally different from prion infection, since the $\mathrm{PrP}^{106-126}$ peptide has never been detected in vivo and is not infectious per se. This raises serious doubts about the relevance and validity of the findings. Another study reported that the neuronal damage was attenuated and prion progression was delayed upon treatment with CSF1 receptor (CSF1R) inhibitor to arrest microglial proliferation, suggesting a detrimental role of microglial proliferation in prion diseases (44).

In an ex vivo model of prion diseases, ganciclovir-mediated microglial depletion in cerebellar organotypic cultured slices from tga20/CD11b-thymidine kinase of herpes simplex virus (tga20/CD11b-HSVTK) transgenic mice resulted in enhanced $\operatorname{PrP}^{\mathrm{sc}}$ deposition and increased prion titer. These results suggest that microglia play an important role in clearing prions $(45,46)$. The same ex vivo model was used to determine the effect of microglial depletion on prion-induced neurodegeneration. After microglial depletion, not only $\mathrm{PrP}^{\mathrm{sc}}$ accumulation but also neurotoxicity of prions were drastically enhanced, and prion pathology was markedly aggravated (47). Moreover, while the tga20/ CD11b-HSVTK transgenic mice were intracerebrally infected with prions, followed by implantation of osmotic minipumps containing ganciclovir to deliver the drug directly into the brain, the incubation time of prion-infected microglia-depleted mice was significantly shortened (47). Additionally, when prions were inoculated into Il34 ${ }^{-/}$mice, which contain fewer microglia because of impaired microglial development and maintenance $(48,49)$, $\mathrm{PrP}^{\mathrm{sc}}$ deposition was augmented and prion progression was accelerated in comparison with infected WT littermates (47). Therefore, we contend that microglia play a generally neuroprotective rather than deleterious role in prion pathogenesis (Figure 2). Neuroprotection by microglia is mediated, at least partly, by prion 
Table 1. Role of microglia-related molecules in prion pathogenesis in the CNS

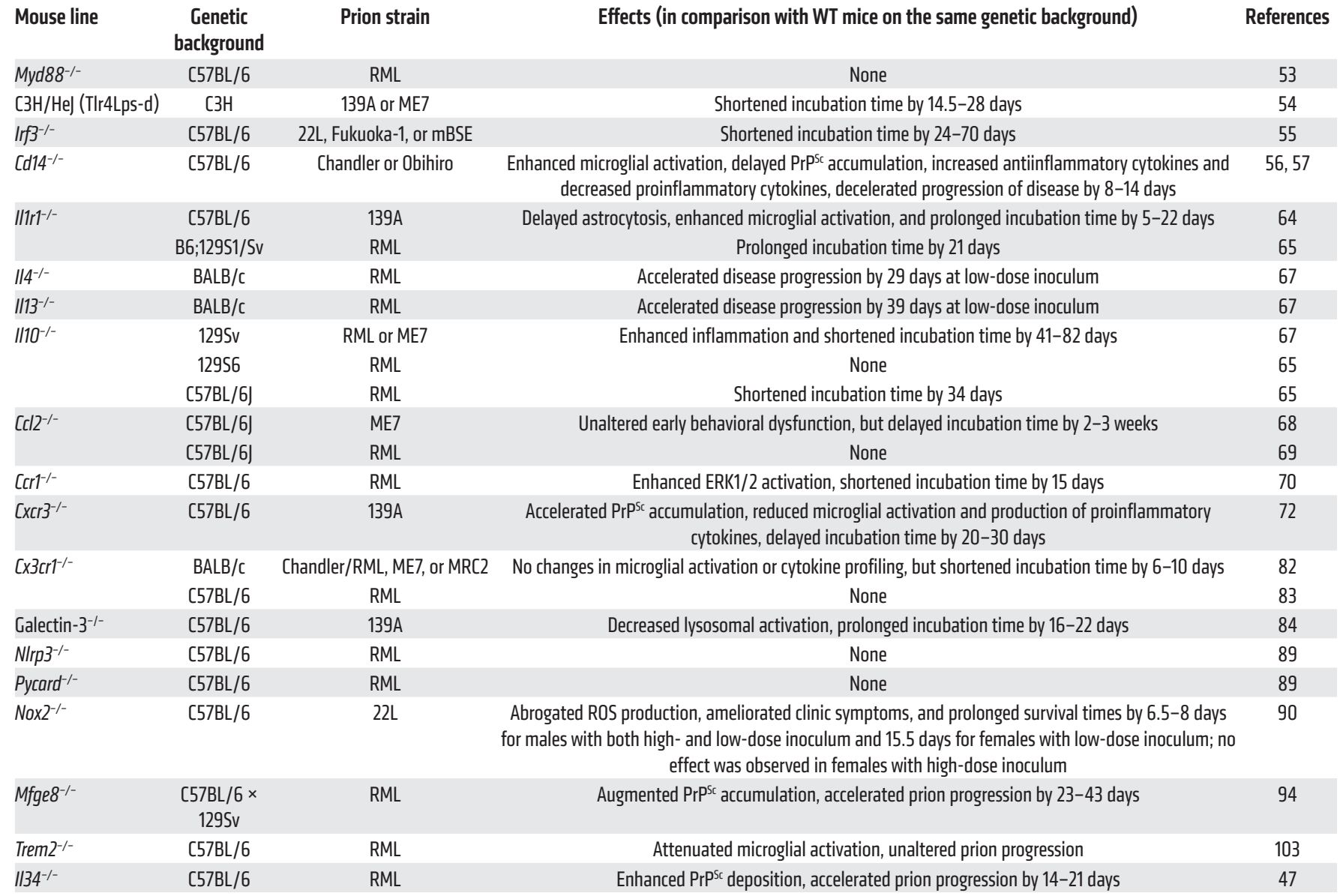

clearance. These results also suggest that prion dissemination in the CNS is not executed by microglia. In line with this conclusion, when prion-infected mice were subjected to an additional intraperitoneal infection of Moloney murine leukemia virus, which can invade the brain, an enhanced microglial activation associated with transient clearance and delayed accumulation of prions in the brain were observed (50).

\section{Microglia-related transducers in prion diseases}

Toll-like receptors. As discussed above, prion progression is accompanied by conspicuous microglial activation; however, the molecular mechanisms underlying the microglial response to prion infection are not well defined. Toll-like receptors (TLRs) are a class of pattern-recognition receptors that recognize invading pathogens to initiate the innate immune response. Therefore, TLRs are potential candidates involved in prion-induced microglial activation to defend against prion infection.

An early study reported that repeated stimulation of TLR9 by unmethylated $\mathrm{CpG}$ oligodinucleotides was protective in mice peripherally inoculated with prions, resulting in delay or prevention of disease (51). This protection was revealed to be due to alterations in the morphology and function of peripheral lymphoid structures (52). Mice that lack the adaptor protein MyD88 (Myd88 ${ }^{-/-}$mice), which are defective in most TLR signaling and IL-1 receptor (IL-1R) signaling, were challenged intraperitoneally or intracerebrally with the standard Rocky Mountain Laboratory (RML) strain of prions.

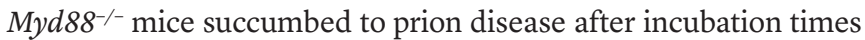
similar to those of WT mice (Table 1), suggesting that Myd88dependent TLR signaling or IL-1R signaling does not play a major role in prion pathogenesis (53). Nevertheless, another study observed a significantly shortened incubation time in $\mathrm{C} 3 \mathrm{H} / \mathrm{HeJ}$ (Tlr4Lps-d) mice, which possess a mutation in the TLR4 intracellular domain that prevents TLR4 signaling, compared with the strain-matched WT control $(\mathrm{C} 3 \mathrm{H} / \mathrm{HeOuJ})$. Therefore, stimulation of TLR4, perhaps via a Myd88-independent pathway, may play a protective role in prion pathogenesis (54). In accordance with this observation, mice deficient in IFN regulatory factor 3 ( $\mathrm{Irf3}^{-1-}$ ), a key transcription factor of the MyD88-independent type I IFN production pathway, showed a significantly earlier onset of prion pathology than WT controls. In addition, overexpression of IRF3 in a cell culture system attenuated prion infection, whereas prion-infected cells treated with IRF3-targeted siRNAs accumulated more PrPsc 
(55). These results indicate that IRF3 negatively regulates $\mathrm{PrP}^{\mathrm{Sc}}$ formation and prion pathogenesis, providing new insight into the role of the innate immune system in the response to prion infection. Overall, these results suggest a general protective role of certain TLR signaling pathways in prion pathogenesis. Since TLRs are expressed by various cell types in the CNS, microglia-specific manipulation of TLRs (such as by Cx3cr1-Cre-dependent conditional knockout of TLRs) is required to further determine the role of microglial TLRs in prion pathogenesis.

CD14 is a glycosylphosphatidylinositol-anchored (GPI-anchored) protein mainly expressed by microglia in the CNS. CD14 acts as a TLR4 coreceptor to recognize LPS. Upregulation of CD14 expression associated with aggregated proteins has been observed in both patients and mouse models of neurodegenerative disorders including prion diseases. Upon intracerebral challenge with prions, $C d 14^{-/-}$mice survived longer than WT mice, suggesting that CD14 contributes to prion disease progression. Quantification of $\operatorname{PrP}^{\mathrm{Sc}}$ revealed that accumulation of $\mathrm{PrP}^{\mathrm{Sc}}$ was delayed in $\mathrm{Cd} 14^{-/-}$mice compared with WT controls. This is associated with enhanced microglial activation in prion-infected $C d 14^{-/-}$mice. Moreover, the microglia-derived antiinflammatory cytokines IL-10 and TGF- $\beta$ and neuron-derived IL-13 appeared to be expressed earlier in prion-infected $C d 14^{-/-}$mice. In contrast, expression of the proinflammatory cytokine IL-1 $\beta$ in microglia appeared to be decreased in $\mathrm{Cd} 14^{-/-}$mice in the early stage of prion infection. These results imply that CD14 plays a detrimental role in prion pathogenesis by promoting proinflammatory responses and/or inhibiting antiinflammatory responses in the brain $(56,57)$.

Complement systems. As an integral part of the innate immune system, the complement system provides an important immune-mediated defense against pathogens. We have long held the view that complement facilitates prion pathogenesis by trapping and retaining prions in secondary lymphoid organs after peripheral infection $(58,59)$. The complement cascade was reported to be activated in the brains of patients with Creutzfeldt-Jakob disease and Gerstmann-Sträussler-Scheinker disease, as well as in animal models of prion infection (60-62). Overall, complement activity was upregulated in several prion-infected rodent brains in a timedependent manner, and complement components such as C1q, C3, and membrane attack complex (MAC) were also upregulated and detected in various cell types, including microglia (62). Nevertheless, the contribution of the complement system in prion-induced neurodegeneration in the CNS seems to be minor, as mice deficient in various complement components developed disease similarly to WT mice upon intracerebral challenge with prions $(58,59,63)$.

Cytokines. As discussed above, microglial activation in prion disease is typically associated with increased production of cytokines. The involvement of cytokines in prion pathogenesis was elucidated by challenging of different mouse models lacking or overexpressing specific cytokines with prions. While most cytokines (TNF- $\alpha$, IL-6, TGF- $\beta 1$, etc.) do not play an important role in prion-induced neurodegeneration in the CNS, knockout of IL-1 receptor 1 (IL-1R1), which is the receptor for IL-1 $\alpha$ and IL-1 $\beta$, led to a modest but statistically significant delay in disease progression $(64,65)$. This may be due to delayed astrogliosis and/or augmented microglial activation that contribute to enhanced phagocytosis of prions in $I l 1 \mathrm{rI}^{-/-}$mice. Additionally, an association study showed a correlation between SNPs in the Il1r1 locus and incubation time in mouse models of prion diseases, supporting the involvement of IL-1R1 signaling in prion pathogenesis (66).

Several antiinflammatory cytokines have been examined for their contribution to prion disease. While deficiency in IL-4 or IL-13 had minor or negligible effects on prion disease progression, deficiency in IL-10 on a $129 \mathrm{~Sv}$ background markedly shortened time between inoculation and onset of clinical symptoms (67). In the absence of IL-10, expression of the proinflammatory cytokine TNF- $\alpha$ was enhanced at an early disease stage, which could account for the enhanced prion pathology in $\mathrm{IlO}^{-/-}$mice. This study suggests that the antiinflammatory cytokine IL-10 has a neuroprotective role in prion pathogenesis. Interestingly, the neuroprotective role of IL-10 in prion disease seems to depend on the genetic context, since another study observed only a mild acceleration of prion progression in $\mathrm{Il1O}^{-/-}$mice on a C57BL/6 background but not on a 129S1/SvImj background (65).

Chemokines. Most neurodegenerative disorders, including prion diseases, are associated with upregulation of chemokine expression. For instance, expression of the chemokine ligand CCL2 (also known as monocyte chemoattractant protein-1, MCP-1), is coordinately upregulated in the CNS with disease progression in C57BL/6 mice infected with ME7 prions (68). The increased expression of CCL2 also positively correlated with microglial activation. Following inoculation of ME7 prions, $\mathrm{Ccl}^{-1-}$ mice experienced microglial activation or neuronal death in the hippocampus and thalamus at levels similar to those of WT controls, but disease incubation time was significantly prolonged by CCL2 deficiency (68). Interestingly, the effect of CCL2 on prion pathogenesis might be prion strain-dependent, since $\mathrm{Ccl} 2^{--}$ mice failed to show any changes in disease progression after inoculation of RML prions (69). Mice lacking the CCL2 receptor CCR2 displayed similar disease progression to WT mice following RML prion challenge $(21,65)$. However, CCR2 deficiency blocked recruitment of perivascular macrophages in the diseased CNS and resulted in a mild reduction in microglia proliferation (21).

Expression of CCL5 (also known as RANTES) and its receptors CCR1, CCR3, and CCR5 is also increased in mouse models of prion diseases (36). CCR1 deficiency resulted in augmented induction of CCR5 and CCR3, probably by a compensatory mechanism, leading to enhanced activation of ERK1/2 and accelerated disease progression in prion-infected mice (70). However, mice lacking CCR5 developed prion disease at a rate similar to that of WT controls following RML prion inoculation (65). Thus, the exact role of these chemokine and chemokine receptors in prion pathogenesis remains unclear.

CXCL9 and CXCL10, which recognize and bind to CXCR3, are also increased in prion infection $(64,71)$. After intracerebral inoculation of $139 \mathrm{~A}$ prion, $\mathrm{Cxcr3}^{-/-}$mice accumulated more $\mathrm{PrP}^{\mathrm{Sc}}$ in their brains compared with WT mice, but succumbed to disease with a prolonged incubation time (72). Further characterization of these mice revealed that absence of CXCR3 reduced microglial activation and consequently impaired prion phagocytosis and degradation, leading to enhanced $\mathrm{PrP}^{\mathrm{Sc}}$ accumulation. However, depletion of CXCR3 resulted in reduced production of proinflammatory cytokines, which could explain the prolonged incubation time. The chemokine CXCL13 is also upregulated in prion disease $(42,71)$; however, knockout of its receptor, CXCR5, did not alter prion pathogenesis after intracerebral inoculation of RML prions (73). 
CX3CR1, the receptor of the neuron-expressed chemokine CX3CL1 (also known as fractalkine), is exclusively expressed by microglia in the CNS $(74,75)$. CX3CL1/CX3CR1 signaling represents one example of the neuron-microglia crosstalk that maintains microglia in a quiescent state (76). CX3CR1 has been extensively studied in several models of neurodegeneration, such as Alzheimer's disease (AD), Parkinson's disease, and amyotrophic lateral sclerosis; however, conflicting results have been reported in these models (77-80). In animal models of prion infection, the CX3CL1/CX3CR1 axis has been reported to be altered $(75,81)$. The role of CX3CR1 in prion disease has been studied by two independent groups. While one group demonstrated that lack of CX3CR1 led to modest but significant reductions in incubation time (82), suggesting a neuroprotective role of CX3CR1 signaling in prion pathogenesis, the other group failed to find any difference in disease onset, duration of disease, or pathology of disease between the $\mathrm{C} \times 3 \mathrm{crI}^{-/-}$mice and WT controls (83). The discrepancy between these two studies may be due to the distinct mouse strains or different experimental protocols used in each study. Nevertheless, neither study observed any obvious effect of CX3CR1 on prion-induced microglial activation. Moreover, these studies suggest an overall minor effect of CX3CR1 on prion pathogenesis. Therefore, as in other neurodegeneration models, the role of CX3CR1 in prion disease may vary with different experimental systems.

Inflammation regulators. Galectin-3 is a multifunctional protein and, in particular, a regulator of inflammation. Immunofluorescence staining identified microglia as the major source of galectin-3 in the brain. Expression of galectin-3 is significantly upregulated in prion-infected brains. Upon prion infection, galectin-3 deficiency did not affect $\mathrm{PrP}^{\mathrm{Sc}}$ deposition or the development of gliosis. However, galectin- $3^{-/-}$mice showed prolonged survival times after prion infection. Interestingly, expression of the lysosomal activation marker LAMP-2 was profoundly decreased in prion-infected galectin- $3^{-/-}$mice, suggesting that galectin-3 is involved in regulation of lysosomal function. These results suggest a detrimental role of galectin-3 in prion pathogenesis in the CNS and indicate that lysosomal dysfunction may contribute to prion disease progression (84).

The inflammasome is a cytosolic multiprotein complex that serves as a scaffold for activating the proinflammatory cytokines IL-1 $\beta$ and IL-18 via caspase-1-mediated cleavage. The NLRP3 inflammasome has been reported to be activated in AD and contributes to pathology in a mouse model of $\operatorname{AD}(85,86)$. In in vitro cell culture models using microglia cell lines or primary murine microglia, the NLRP3 inflammasome was found to be essential for IL-1 $\beta$ release upon treatment with $\mathrm{PrP}^{106-126}$ peptide (87) or PrP fibrils (88). However, whether the NLRP3 inflammasome can recognize bona fide prions and affect prion pathogenesis in vivo was unknown. The role of the NLRP3 inflammasome in mouse models of prion disease was assessed by intracerebral inoculation of RML prions into mice deficient in NLRP3 $\left(\mathrm{Nlrp3}^{-/-}\right)$or the inflammasome adaptor protein ASC (Pycard ${ }^{-/-}$. Both Nlrp3 $3^{-/-}$and Pycard ${ }^{-/-}$mice developed the classic symptoms of prion diseases and succumbed to scrapie with attack rates and survival times similar to those of WT controls. Additionally, the absence of NLRP3 or ASC did not significantly change levels of IL-1 $\beta$ in the brain at the terminal stage of the disease. Thus, based on existing data, NLRP3 and ASC do not play significant roles in prion pathogenesis (89).

Reactive oxygen species and NOX2. Prion-induced neurodegeneration is often accompanied by the production of reactive oxygen species (ROS) and oxidative stress. Indeed, NOX2, a membrane-bound electrochemical pump and a prominent NADPH oxidase that generates ROS, is markedly upregulated in microglia within affected brain regions of patients with Creutzfeldt-Jakob disease, as well as in prion-inoculated mouse brains and cerebellar organotypic cultured slices. Upon challenge with prions, NOX2-deficient mice showed delayed onset of motor deficits and a significant prolongation of incubation time compared with WT mice. Dihydroethidium assays demonstrated a marked ROS burst at the terminal stage of disease in WT mice, but not in NOX2-deficient mice. Thus, NOX2 is a major contributor to excessive production of ROS in prion diseases and therefore affects prion pathogenesis (90).

Phagocytosis mediators. Milk fat globule epidermal growth factor 8 (MFGE8) is a bifunctional secreted molecule that can bind to both phosphatidylserine on apoptotic cells and integrins on phagocytes, thereby bridging apoptotic bodies and phagocytes and facilitating phagocytosis (91). Because prion infectivity is usually associated with lipids $(92,93)$, it is conceivable that MFGE8 is involved in prion clearance by microglia in prion-infected brains. Indeed, mice lacking MFGE8 ( $\mathrm{Mfge}^{-\mathrm{-}^{-}}$) experienced augmented prion accumulation and accelerated disease progression after prion inoculation. Interestingly, the involvement of MFGE8 in microglia-mediated prion clearance is mouse strain-dependent, since the effect of MFGE8 depletion was observed in mice with C57BL/6 ' 129Sv background but not C57BL/6 background (94). This finding also implies the existence of further polymorphic determinants of prion clearance.

Triggering receptor expressed on myeloid cells 2 (TREM2) is an innate immune cell surface receptor mainly expressed by microglia in the brain. TREM2 facilitates phagocytosis of apoptotic neurons and bacteria, thereby suppressing inflammation $(95,96)$. Dysfunctional variants of TREM2 have been identified as major genetic risk factors for $\mathrm{AD}$ and other neurodegenerative conditions (97-101). Ablation of Trem2 in a mouse model of $\mathrm{AD}(5 \mathrm{xFAD})$ resulted in increased hippocampal $\mathrm{A} \beta$ burden and accelerated loss of cortical neurons. Interestingly, microglial activation was markedly attenuated in 5xFAD mice on a Trem $2^{-/}$background (102). To explore whether TREM2 is also involved in prion clearance by microglia, Trem $2^{-/-}$mice and their WT littermates were intracerebrally inoculated with prions. Neither $\mathrm{PrP}^{\mathrm{Sc}}$ deposition nor disease progression was different between Trem $2^{-/-}$and WT mice, suggesting that TREM2 is not a major player in prion clearance. Nevertheless, similarly to what was observed in $5 \mathrm{xFAD}$ model, markers of microglial activation (IBA1) were significantly reduced in prion-infected Trem $2^{-/-}$mice, suggesting that TREM2 is involved in prioninduced microglial activation (103).

\section{Therapeutics of prion disease by targeting of microglia}

Microglia play an overall neuroprotective role in prion diseases and are therefore considered as friend rather than foe. Thera- 
peutic strategies against prion disease should be designed to avoid depleting or reducing microglia. However, microglia that are activated upon prion infection are heterogeneous and can adopt different phenotypes. Whereas some activated microglia possess enhanced phagocytosis activity and produce antiinflammatory factors, thereby exerting neuroprotective functions, others could be more proinflammatory and neurotoxic in the brain. Accordingly, it is conceivable that microglia could be guided toward a beneficial state that could efficiently phagocytose prions and secrete antiinflammatory factors to slow down disease progression.

Several microglia-related molecules and pathways are involved in prion-induced microglial activation and prion pathogenesis. Attempts to modulate prion progression by blocking TLRs via administration of unmethylated CpG oligodinucleotides caused a delay in prion progression in mice peripherally inoculated with prions (51). However, this effect may have been unrelated to microglia and instead mainly due to changes in the morphology and function of peripheral lymphoid elements (52). CD14 deficiency confers neuroprotection from prion disease by modifying cytokine secretion. Treatment with the GPI-specific phospholipase glimepiride could induce shedding of CD14 from the cell surface, thereby modifying prion progression (104).

CSF1R signaling is one of the mediators of prion-induced microglial activation and proliferation. Treatment with the selective CSF1R tyrosine kinase inhibitor GW2580 significantly reduced microglial proliferation in ME7-infected mice. In addition, there was decreased expression of proinflammatory cytokines (IL- 6 and IL-1 $\beta$ ) and increased expression of antiinflammatory cytokines (ARG1 and YM1). Blocking of CSF1R by GW2580 also improved behavioral performance and prolonged survival of prion-infected mice (44). Therefore, manipulation of microglial activation to a beneficial phenotype represents a potential therapeutic approach for prion disease.

Microglia exert neuroprotection in prion disease, at least partly by engulfing and degrading prions; however, phagocytosis and the clearance of prions are inefficient, and animals eventually die of the disease (105). This is probably because the early phagocytic and antiinflammatory microglia phenotypically switch to a proinflammatory and less phagocytic phenotype at the late stage of disease. Strategies aiming to enhance phagocytic capacity of microglia have been explored. Retrovirus treatment of prion-infected mice transiently stimulated microglial activation and prion clearance, although the incubation time was not altered (50). These results suggest that appropriate stimulation of microglial activation could be beneficial in prion disease. However, stimulation of microglia should be carefully controlled, since intracerebral or systemic administration of LPS to ME7-infected mice markedly stimulated microglial activation, but exacerbated the local inflammatory response and increased neuronal death (106). Intracerebral injection of brain-engraftable murine microglial cells that express an antiPrP single-chain variable fragment before or at an early stage after prion infection modestly but significantly prolonged the incubation times, suggesting a novel microglia-mediated immunotherapeutic approach against prion diseases (107).

\section{Perspectives}

Microglial activation is now widely recognized in most neurodegenerative disorders, including prion diseases. Several models of microglial depletion and deficiency provide convergent evidence of a neuroprotective role of microglia in prion pathogenesis. However, the exact molecular mechanisms underlying the microglial response to prion infection remain to be fully elucidated. Moreover, therapeutic strategies that modulate the effects of microglia in prion pathogenesis are challenging because of the heterogeneity of activated microglia. Characterization of the different microglial populations represents a crucial step toward a deeper understanding of microglial activation in prion pathogenesis.

Under physiological conditions, CNS microglia undergo complex and sometimes subtle interactions with other cell types such as neurons or astrocytes to elicit various functions. Upon prion infection, microglial activation is usually associated with astrogliosis. Previous findings indicate that the astrocytederived phagocytosis mediator MFGE8 enhances phagocytosis of prions by microglia, thus providing an important cross-talk between different cell types during prion infection (94). However, the mechanisms by which microglial activation and astrocytes orchestrate their response to prions remain unclear. Classically activated microglia have been found to induce a putative subtype of reactive astrocytes, termed A1, by secreting IL- $1 \alpha$, TNF- $\alpha$, and C1q, leading to a loss of neurotrophic functions and neuronal death (108). Thus, microglial activation and astrogliosis may be more closely related than has previously been appreciated. Because of the heterogeneity and diversity of astrocyte subtypes in the CNS (109), the complex interaction of microglia and astrocytes requires further investigation.

A deeper understanding of the role of microglia in prion pathogenesis may facilitate the development of techniques to manipulate microglial activation and dampen disease progression. Hopefully, microglial activation can be used as a novel target to interfere with prion progression. Since neuroinflammation including microglial activation is a common feature of many neurodegenerative diseases, the study of microglia in prion diseases could reveal important insights into the role of neuroinflammation in other neurodegenerative conditions.

\section{Acknowledgments}

A. Aguzzi is the recipient of an Advanced Grant of the European Research Council (no. 250356) and is supported by grants from the European Union (PRIORITY, NEURINOX), the Swiss National Foundation (including a Sinergia grant), the GELU Foundation, the Swiss Initiative in Systems Biology, SystemsX.ch (PrionX, SynucleiX), and the Klinische Forschungsschwerpunkte "Small RNAs" and "Human Hemato-Lymphatic Diseases." The funders had no role in study design, data collection and analysis, decision to publish, or preparation of the manuscript.

Address correspondence to: Adriano Aguzzi or Caihong Zhu, Institute of Neuropathology, University Hospital Zurich, Schmelzbergstrasse 12, CH-8091 Zurich, Switzerland. Phone: 41.44.255.2108; Email: Adriano.Aguzzi@usz.ch (A. Aguzzi), Caihong.Zhu@usz.ch (C. Zhu). 
1. Aguzzi A, Nuvolone M, Zhu C. The immunobiology of prion diseases. Nat Rev Immunol. 2013;13(12):888-902.

2. Aguzzi A, Zhu C. Five questions on prion diseases. PLoS Pathog. 2012;8(5):e1002651.

3. Gajdusek DC, Zigas V. Degenerative disease of the central nervous system in New Guinea; the endemic occurrence of kuru in the native population. N Engl J Med. 1957;257(20):974-978.

4. Wells GA, et al. A novel progressive spongiform encephalopathy in cattle. Vet Rec. 1987;121(18):419-420.

5. Will RG, et al. A new variant of Creutzfeldt-Jakob disease in the UK. Lancet. 1996;347(9006):921-925.

6. Prusiner SB. Novel proteinaceous infectious particles cause scrapie. Science. 1982;216(4542):136-144.

7. Alliot F, Godin I, Pessac B. Microglia derive from progenitors, originating from the yolk sac, and which proliferate in the brain. Brain Res Dev Brain Res. 1999;117(2):145-152.

8. Ginhoux F, et al. Fate mapping analysis reveals that adult microglia derive from primitive macrophages. Science. 2010;330(6005):841-845

9. Schulz C, et al. A lineage of myeloid cells independent of Myb and hematopoietic stem cells. Science. 2012;336(6077):86-90.

10. Kierdorf K, et al. Microglia emerge from erythromyeloid precursors via Pu.1- and Irf8-dependent pathways. Nat Neurosci. 2013;16(3):273-280.

11. Matcovitch-Natan O, et al. Microglia development follows a stepwise program to regulate brain homeostasis. Science. 2016;353(6301):aad8670.

12. Chen SK, et al. Hematopoietic origin of pathological grooming in Hoxb8 mutant mice. Cell. 2010;141(5):775-785.

13. Paolicelli RC, et al. Synaptic pruning by microglia is necessary for normal brain development. Science. 2011;333(6048):1456-1458.

14. Parkhurst CN, et al. Microglia promote learningdependent synapse formation through brain-derived neurotrophic factor. Cell. 2013;155(7):1596-1609.

15. Nimmerjahn A, Kirchhoff F, Helmchen F. Resting microglial cells are highly dynamic surveillants of brain parenchyma in vivo. Science. 2005;308(5726):1314-1318.

16. Aguzzi A, Barres BA, Bennett ML. Microglia: scapegoat, saboteur, or something else? Science. 2013;339(6116):156-161.

17. Hanisch UK, Kettenmann H. Microglia: active sensor and versatile effector cells in the normal and pathologic brain. Nat Neurosci. 2007;10(11):1387-1394.

18. Block ML, Zecca L, Hong JS. Microglia-mediated neurotoxicity: uncovering the molecular mechanisms. Nat Rev Neurosci. 2007;8(1):57-69.

19. Cherry JD, Olschowka JA, O’Banion MK. Neuroinflammation and M2 microglia: the good, the bad, and the inflamed. J Neuroinflammation. 2014;11:98.

20. Heneka MT, Kummer MP, Latz E. Innate immune activation in neurodegenerative disease. Nat Rev Immunol. 2014;14(7):463-477.

21. Gómez-Nicola D, Schetters ST, Perry VH. Differential role of CCR2 in the dynamics of microglia and perivascular macrophages during prion disease. Glia. 2014;62(7):1041-1052.

22. Miyazono M, Iwaki T, Kitamoto T, Kaneko Y, Doh-ura K, Tateishi J. A comparative immunohistochemical study of Kuru and senile plaques with a special reference to glial reactions at various stages of amyloid plaque formation. Am J Pathol. 1991;139(3):589-598.

23. Barcikowska M, Liberski PP, Boellaard JW, Brown $\mathrm{P}$, Gajdusek DC, Budka H. Microglia is a component of the prion protein amyloid plaque in the Gerstmann-Sträussler-Scheinker syndrome. Acta Neuropathol.1993;85(6):623-627.

24. Guiroy DC, Wakayama I, Liberski PP, Gajdusek DC. Relationship of microglia and scrapie amyloid-immunoreactive plaques in kuru, Creutzfeldt-Jakob disease and Gerstmann-Sträussler syndrome. Acta Neuropathol. 1994;87(5):526-530.

25. Sasaki A, Hirato J, Nakazato Y. Immunohistochemical study of microglia in the CreutzfeldtJakob diseased brain. Acta Neuropathol. 1993;86(4):337-344

26. Williams AE, Lawson LJ, Perry VH, Fraser H. Characterization of the microglial response in murine scrapie. Neuropathol Appl Neurobiol. 1994;20(1):47-55

27. Betmouni S, Perry VH, Gordon JL. Evidence fo an early inflammatory response in the central nervous system of mice with scrapie. Neuroscience. 1996;74(1):1-5.

28. Williams A, Lucassen PJ, Ritchie D, Bruce M. PrP deposition, microglial activation, and neuronal apoptosis in murine scrapie. Exp Neurol. 1997;144(2):433-438

29. Giese A, Brown DR, Groschup MH, Feldmann C, Haist I, Kretzschmar HA. Role of microglia in neuronal cell death in prion disease. Brain Pathol. 1998;8(3):449-457.

30. Shi Q, et al. Brain microglia were activated in sporadic CJD but almost unchanged in fatal familial insomnia and G114V genetic CJD. Virol J 2013;10:216.

31. Campbell IL, Eddleston M, Kemper P, Oldstone MB, Hobbs MV. Activation of cerebral cytokine gene expression and its correlation with onset of reactive astrocyte and acute-phase response gene expression in scrapie. J Virol. 1994;68(4):2383-2387.

32. Kordek R, Nerurkar VR, Liberski PP, Isaacson S, Yanagihara R, Gajdusek DC. Heightened expression of tumor necrosis factor alpha, interleukin 1 alpha, and glial fibrillary acidic protein in experimental Creutzfeldt-Jakob disease in mice. Proc Natl Acad Sci U S A. 1996;93(18):9754-9758.

33. Sharief MK, Green A, Dick JP, Gawler J, Thompson EJ. Heightened intrathecal release of proinflammatory cytokines in Creutzfeldt-Jakob disease. Neurology. 1999;52(6):1289-1291.

34. Williams AE, van Dam AM, Man-A-Hing WK, Berkenbosch F, Eikelenboom P, Fraser H. Cytokines, prostaglandins and lipocortin-1 are present in the brains of scrapie-infected mice. Brain Res. 1994;654(2):200-206.

35. Walsh DT, Betmouni S, Perry VH. Absence of detectable IL-1beta production in murine prion disease: a model of chronic neurodegeneration.
J Neuropathol Exp Neurol. 2001;60(2):173-182.

36. Baker CA, Lu ZY, Zaitsev I, Manuelidis L. Microglial activation varies in different models of Creutzfeldt-Jakob disease. J Virol. 1999;73(6):5089-5097.

37. Cunningham C, Boche D, Perry VH. Transforming growth factor beta1, the dominant cytokine in murine prion disease: influence on inflammatory cytokine synthesis and alteration of vascular extracellular matrix. Neuropathol Appl Neurobiol. 2002;28(2):107-119.

38. Stoeck K, et al. Interleukin 4 and interleukin 10 levels are elevated in the cerebrospinal fluid of patients with Creutzfeldt-Jakob disease. Arch Neurol. 2005;62(10):1591-1594.

39. Vincenti JE, et al. Defining the microglia response during the time course of chronic neurodegeneration. J Virol. 2015;90(6):3003-3017.

40. Alibhai J, et al. Distribution of misfolded prion protein seeding activity alone does not predict regions of neurodegeneration. PLoS Biol. 2016;14(11):e1002579.

41. Jeffrey M, Goodsir CM, Bruce ME, McBride PA, Farquhar C. Morphogenesis of amyloid plaques in $87 \mathrm{~V}$ murine scrapie. Neuropathol Appl Neurobiol. 1994;20(6):535-542.

42. Baker CA, Martin D, Manuelidis L. Microglia from Creutzfeldt-Jakob disease-infected brains are infectious and show specific mRNA activation profiles. J Virol. 2002;76(21):10905-10913.

43. Brown DR, Schmidt B, Kretzschmar HA. Role of microglia and host prion protein in neurotoxicity of a prion protein fragment. Nature. 1996;380(6572):345-347

44. Gómez-Nicola D, Fransen NL, Suzzi S, Perry VH. Regulation of microglial proliferation during chronic neurodegeneration. J Neurosci. 2013;33(6):2481-2493.

45. Falsig J, Julius C, Margalith I, Schwarz P, Heppner FL, Aguzzi A. A versatile prion replication assay in organotypic brain slices. Nat Neurosci. 2008;11(1):109-117.

46. Falsig J, Aguzzi A. The prion organotypic slice culture assay - POSCA. Nat Protoc. 2008;3(4):555-562.

47. Zhu C, et al. A neuroprotective role for microglia in prion diseases. JExp Med. 2016;213(6):1047-1059.

48. Wang Y, et al. IL-34 is a tissue-restricted ligand of CSF1R required for the development of Langerhans cells and microglia. Nat Immunol. 2012;13(8):753-760.

49. Greter M, et al. Stroma-derived interleukin-34 controls the development and maintenance of langerhans cells and the maintenance of microglia. Immunity. 2012;37(6):1050-1060.

50. Muth C, et al. Activation of microglia by retroviral infection correlates with transient clearance of prions from the brain but does not change incubation time [published online ahead of print August 25, 2016]. Brain Pathol. https://doi.org/10.1111/bpa.12441.

51. Sethi S, Lipford G, Wagner H, Kretzschmar H. Postexposure prophylaxis against prion disease with a stimulator of innate immunity. Lancet. 2002;360(9328):229-230.

52. Heikenwalder M, et al. Lymphoid follicle destruction and immunosuppression after repeated CpG oligodeoxynucleotide administration. Nat Med. 
2004;10(2):187-192.

53. Prinz M, Heikenwalder M, Schwarz P, Takeda $\mathrm{K}$, Akira S, Aguzzi A. Prion pathogenesis in the absence of Toll-like receptor signalling. EMBO Rep. 2003;4(2):195-199.

54. Spinner DS, et al. Accelerated prion disease pathogenesis in Toll-like receptor 4 signalingmutant mice. JVirol. 2008;82(21):10701-10708.

55. Ishibashi D, et al. Protective role of interferon regulatory factor 3-mediated signaling against prion infection. JVirol. 2012;86(9):4947-4955.

56. Sakai K, et al. Absence of CD14 delays progression of prion diseases accompanied by increased microglial activation. J Virol. 2013;87(24):13433-13445.

57. Hasebe R, Suzuki A, Yamasaki T, Horiuchi M. Temporary upregulation of anti-inflammatory cytokine IL-13 expression in the brains of CD14 deficient mice in the early stage of prion infection. Biochem Biophys Res Commun. 2014;454(1):125-130.

58. Klein MA, et al. Complement facilitates early prion pathogenesis. Nat Med.2001;7(4):488-492.

59. Mabbott NA, Bruce ME, Botto M, Walport MJ, Pepys MB. Temporary depletion of complement component $\mathrm{C} 3$ or genetic deficiency of $\mathrm{C} 1 \mathrm{q}$ significantly delays onset of scrapie. Nat Med. 2001;7(4):485-487.

60. Ishii T, Haga S, Yagishita S, Tateishi J. The presence of complements in amyloid plaques of Creutzfeldt-Jakob disease and GerstmannStraussler-Scheinker disease. Appl Pathol. 1984;2(6):370-379.

61. Kovacs GG, et al. Complement activation in human prion disease. Neurobiol Dis. 2004;15(1):21-28.

62. Lv Y, et al. Remarkable activation of the complement system and aberrant neuronal localization of the membrane attack complex in the brain tissues of scrapie-infected rodents. Mol Neurobiol. 2015;52(3):1165-1179.

63. Mabbott NA, Bruce ME. Complement component C5 is not involved in scrapie pathogenesis. Immunobiology. 2004;209(7):545-549.

64. Schultz J, et al. Role of interleukin-1 in prion disease-associated astrocyte activation. $\mathrm{Am} \mathrm{J}$ Pathol. 2004;165(2):671-678.

65. Tamgüney G, et al. Genes contributing to prion pathogenesis. JGen Virol. 2008;89(pt 7):1777-1788.

66. Akhtar S, et al. Sod1 deficiency reduces incubation time in mouse models of prion disease. PLoS One. 2013;8(1):e54454.

67. Thackray AM, McKenzie AN, Klein MA, Lauder A, Bujdoso R. Accelerated prion disease in the absence of interleukin-10. JVirol. 2004;78(24):13697-13707.

68. Felton LM, Cunningham C, Rankine EL, Waters $\mathrm{S}$, Boche D, Perry VH. MCP-1 and murine prion disease: separation of early behavioural dysfunction from overt clinical disease. Neurobiol Dis. 2005;20(2):283-295.

69. O'Shea M, Maytham EG, Linehan JM, Brandner S, Collinge J, Lloyd SE. Investigation of $\mathrm{mcp} 1$ as a quantitative trait gene for prion disease incubation time in mouse. Genetics. 2008;180(1):559-566.

70. LaCasse RA, Striebel JF, Favara C, Kercher L, Chesebro B. Role of Erk1/2 activation in prion disease pathogenesis: absence of CCR1 leads to increased Erk1/2 activation and accelerated disease progression. J Neuroimmunol. 2008;196(1-2):16-26.

71. Riemer C, Queck I, Simon D, Kurth R, Baier M. Identification of upregulated genes in scrapie-infected brain tissue. J Virol. 2000;74(21):10245-10248.

72. Riemer C, et al. Accelerated prion replication in, but prolonged survival times of, prion-infected CXCR3 ${ }^{-/-}$mice. J Virol. 2008;82(24):12464-12471.

73. Prinz M, et al. Positioning of follicular dendritic cells within the spleen controls prion neuroinvasion. Nature. 2003;425(6961):957-962.

74. Jung S, et al. Analysis of fractalkine receptor CX(3)CR1 function by targeted deletion and green fluorescent protein reporter gene insertion. Mol Cell Biol. 2000;20(11):4106-4114.

75. Hughes PM, Botham MS, Frentzel S, Mir A, Perry VH. Expression of fractalkine (CX3CL1) and its receptor, CX3CR1, during acute and chronic inflammation in the rodent CNS. Glia. 2002;37(4):314-327.

76. Wolf Y, Yona S, Kim KW, Jung S. Microglia, seen from the CX3CR1 angle. Front Cell Neurosci. 2013;7:26.

77. Fuhrmann M, et al. Microglial Cx3cr1 knockout prevents neuron loss in a mouse model of Alzheimer's disease. Nat Neurosci. 2010;13(4):411-413.

78. Lee $S$, et al. CX3CR1 deficiency alters microglial activation and reduces beta-amyloid deposition in two Alzheimer's disease mouse models. Am J Pathol. 2010;177(5):2549-2562.

79. Cardona AE, et al. Control of microglial neurotoxicity by the fractalkine receptor. Nat Neurosci. 2006;9(7):917-924.

80. Bhaskar K, Konerth M, Kokiko-Cochran ON, Cardona A, Ransohoff RM, Lamb BT. Regulation of tau pathology by the microglial fractalkine receptor. Neuron. 2010;68(1):19-31.

81. Xie WL, et al. Abnormal activation of microglia accompanied with disrupted CX3CR1/CX3CL1 pathway in the brains of the hamsters infected with scrapie agent 263K. JMol Neurosci. 2013;51(3):919-932.

82. Grizenkova J, Akhtar S, Brandner S, Collinge J, Lloyd SE. Microglial Cx3cr1 knockout reduces prion disease incubation time in mice. BMC Neurosci. 2014;15:44.

83. Striebel JF, Race B, Carroll JA, Phillips K, Chesebro B. Knockout of fractalkine receptor Cx3cr1 does not alter disease or microglial activation in prion-infected mice. J Gen Virol. 2016;97(6):1481-1487.

84. Mok SW, et al. Role of galectin-3 in prion infections of the CNS. Biochem Biophys Res Commun 2007;359(3):672-678.

85. Halle A, et al. The NALP3 inflammasome is involved in the innate immune response to amyloid-ß. Nat Immunol. 2008;9(8):857-865.

86. Heneka MT, et al. NLRP3 is activated in Alzheimer's disease and contributes to pathology in APP/PS1 mice. Nature. 2013;493(7434):674-678.

87. Shi F, et al. The NALP3 inflammasome is involved in neurotoxic prion peptide-induced microglial activation. J Neuroinflammation. 2012;9:73.
88. Hafner-Bratkovič I, Benčina M, Fitzgerald KA, Golenbock D, Jerala R. NLRP3 inflammasome activation in macrophage cell lines by prion protein fibrils as the source of IL-1 $\beta$ and neuronal toxicity. Cell Mol Life Sci. 2012;69(24):4215-4228.

89. Nuvolone M, Sorce S, Schwarz P, Aguzzi A. Prion pathogenesis in the absence of NLRP3/ASC inflammasomes. PLoS One. 2015;10(2):e0117208

90. Sorce $\mathrm{S}$, et al. The role of the NADPH oxidase NOX2 in prion pathogenesis. PLoS Pathog. 2014;10(12):e1004531.

91. Hanayama R, Tanaka M, Miwa K, Shinohara A, Iwamatsu A, Nagata S. Identification of a factor that links apoptotic cells to phagocytes. Nature. 2002;417(6885):182-187.

92. Klein TR, Kirsch D, Kaufmann R, Riesner D. Prion rods contain small amounts of two host sphingolipids as revealed by thin-layer chromatography and mass spectrometry. Biol Chem. 1998;379(6):655-666.

93. Rouvinski A, et al. Live imaging of prions reveals nascent PrPSc in cell-surface, raftassociated amyloid strings and webs. JCell Biol. 2014;204(3):423-441.

94. Kranich J, et al. Engulfment of cerebral apoptotic bodies controls the course of prion disease in a mouse strain-dependent manner. JExp Med. 2010;207(10):2271-2281.

95. N'Diaye EN, et al. TREM-2 (triggering receptor expressed on myeloid cells 2 ) is a phagocytic receptor for bacteria. JCell Biol. 2009;184(2):215-223.

96. Takahashi K, Rochford CD, Neumann H. Clearance of apoptotic neurons without inflammation by microglial triggering receptor expressed on myeloid cells-2. J Exp Med. 2005;201(4):647-657.

97. Guerreiro R, et al. TREM2 variants in Alzheimer's disease. N Engl J Med. 2013;368(2):117-127.

98. Jonsson T, et al. Variant of TREM2 associated with the risk of Alzheimer's disease. $N$ EnglJMed. 2013;368(2):107-116.

99. Rayaprolu S, et al. TREM2 in neurodegeneration: evidence for association of the p.R $47 \mathrm{H}$ variant with frontotemporal dementia and Parkinson's disease. Mol Neurodegener. 2013;8:19.

100.Guerreiro RJ, et al. Using exome sequencing to reveal mutations in TREM2 presenting as a frontotemporal dementia-like syndrome without bone involvement. JAMA Neurol. 2013;70(1):78-84.

101.Cady J, et al. TREM2 variant p.R47H as a risk factor for sporadic amyotrophic lateral sclerosis. JAMA Neurol. 2014;71(4):449-453.

102. Wang $Y$, et al. TREM2 lipid sensing sustains the microglial response in an Alzheimer's disease model. Cell. 2015;160(6):1061-1071.

103. Zhu C, et al. Triggering receptor expressed on myeloid cells- 2 is involved in prion-induced microglial activation but does not contribute to prion pathogenesis in mouse brains. Neurobiol Aging. 2015;36(5):1994-2003.

104.Ingham V, Williams A, Bate C. Glimepiride reduces CD14 expression and cytokine secretion from macrophages. J Neuroinflammation. 2014;11:115.

105. Hughes MM, Field RH, Perry VH, Murray CL, Cunningham C. Microglia in the degenerating 
brain are capable of phagocytosis of beads and of apoptotic cells, but do not efficiently remove PrPSc, even upon LPS stimulation. Glia. 2010;58(16):2017-2030.

106.Cunningham C, Wilcockson DC, Campion S, Lunnon K, Perry VH. Central and systemic endotoxin challenges exacerbate the local inflam- matory response and increase neuronal death during chronic neurodegeneration. J Neurosci. 2005;25(40):9275-9284.

107. Fujita K, et al. Effects of a brain-engraftable microglial cell line expressing anti-prion scFv antibodies on survival times of mice infected with scrapie prions. Cell Mol Neurobiol.
2011;31(7):999-1008.

108. Liddelow SA, et al. Neurotoxic reactive astrocytes are induced by activated microglia. Nature. 2017;541(7638):481-487.

109. John Lin CC, et al. Identification of diverse astrocyte populations and their malignant analogs. Nat Neurosci. 2017;20(3):396-405. 